\title{
Contemporary treatment of salivary gland tumors. A review of the literature
}

\author{
loanna Polichroniadou', Panagiotis Karakostas ${ }^{2}$, \\ Svetoslav Slavkov ${ }^{3}$, Assya Krasteva ${ }^{4}$
}

1. Faculty of Dental Medicine, Medical University Sofia, Bulgaria;

2. Department of Preventive Dentistry, Periodontology and Implant Biology, School of Dentistry, Aristotle University - Thessaloniki, Greece;

3. University Specialized Hospital for Maxillofacial Surgery, Medical University of Sofia, Bulgaria. Department of Imaging and Oral Diagnostic, Faculty of Dental Medicine, Medical University - Sofia, Bulgaria.

\begin{abstract}
Aim: The forthcoming study focuses on the opportunities of different treatment related to salivary gland tumors as well as some intraoperative dangers how the latter should be managed.

Materials and Methods: A thorough study of the English literature of the electronic databases "Pubmed", "Scopus" and "Google Scholar" was held in order to accomplish our review properly.

Results: A great variety of surgical techniques and therapeutic approaches are reported while the most important ones are described thoroughly. Moreover, adverse events and recurrence are directly related to surgeon's experience and substantial practice.

Conclusion: Extracapsular dissection constitutes the treatment of choice due to its significant lower incidence of adverse events as a less invasive surgical approach. In carefully selected patients, an
\end{abstract}


application of surgical approach and combined radiotherapy and chemotherapy postoperatively may lead to improved outcomes.

\section{Keywords: contemporary treatment, salivary gland neoplasms, salivary gland tumors}

\section{Background}

Salivary gland tumors constitute a great number of pathologic situations related to the anatomic site of the gland (1). These neoplasms comprise approximately $40 \%$ of all parapharyngeal space tumors (2). The current classification of salivary gland tumors includes five main categories as well as two subcategories depending on the extension of the lesion (Table 1) (3).

In the first half of 20th century, enucleation was referred as the treatment of choice for benign parotid swellings leading at most cases in spillage of the lesion and increased recurrence percentages; as regards pleomorphic adenoma it was detected with $45 \%$ recurrence rate (4).

In the second half of the 20th century enucleation was replaced with superficial parotidectomy (SP), especially in cases of parotid neoplasms deriving from the superficial layer of the glands been taught and practiced as the standard management for most parotid tumors arising in the superficial part of the gland (4). Although this therapeutic approach led in less than 5\% recurrence rate, it was inculpated for a number of adverse events, including facial hollowing, Frey's syndrome, damage of the great auricular nerve and branches of the facial nerve itself[4]. Meanwhile, a number of surgeons gradually started to recommend less invasive surgical approach for the excision of non malignant neoplasms as a way to decrease not only morbidity but also complication rates (4).

In late 1990s extracapsular dissection (ECD) was introduced as an alternative technique, instead of SP in presence of mobile, non malignant parotid swellings, followed by a lower risk of facial nerve damage or Frey's syndrome presence (4). As a result, SP is now conducted in a very limited range of cases (4).

\section{Review results}

Diagnosis of salivary gland tumors is set with the use of fine needle aspiration cytology and core biopsy, open biopsy, frozen section and imaging methods including ultrasound, computed tomography and magnetic resonance imaging (3).

The treatment of the aforementioned neoplasms has been a field of great interest leading in a significant range of different approaches, including surgery, neck surgery, radiotherapy and chemotherapy (Table 2) $(5,6)$. In general, both location and gland involved affect the choice of the appropriate surgical approach (1). 


\section{Total parotidectomy.}

Total parotidectomy constitutes the removal of all parotid gland lateral and medial to the facial nerve with the use of the modified Blair incision[7]. Nevertheless, this treatment is invasive and there is a high risk of facial scars and facial nerve injury (1).

\section{Superficial parotidectomy}

SP divides smaller part of facial nerve and excises at least 2-cm cuff (except when the tumor lies nearby the facial nerve) of surrounding parotid tissue without sacrificing healthy parotid tissue distant from the lesion (7). This approach is indicated in cases of less than $4 \mathrm{~cm}$ benign or low-grade, mobile malignant lesions (7). SP is a common treatment procedure for pleomorphic adenoma, while transient facial nerve palsy may be an adverse event (1).

\section{Trancervical approach}

This surgical procedure is conducted when deep lobe parotid neoplasms are present and have been expanded into the parapharyngeal space (2). Trancervical approach is not recommended unless cervical lymph node metastasis is clinically evident (8). During the surgery, facial nerve is preserved whereas the parotid parenchyma is separated, so as to ensure excision of the lesion in toto and inadvertent facial nerve damage (2). However, is not rare the presence of first bite syndrome after such operations (2). This syndrome occurs initially after an oral intake and it is characterized by recurrent, severe pain nearby parotid which decreases gradually (2). The syndrome may be a result of sacrificing the sympathetic chain or during the removal of deep lobe parotid swelling (2). Severe symptoms can be handled either with drug medication or with botulinum toxin injection (2).

\section{Rhytidectomy}

Rhytidectomy constitutes a different type of incision which similar access compared to parotidectomy in general, and a more favorable postoperative aesthetic appearance (1).

\section{Extracapsular dissection}

ECD constitutes the most indicative treatment since it is characterized by facial nerve prevention, meticulous hemostasis and minimal invasive approach (hidden as a face lift technique) resulting in the removal of merely a small cuff of healthy parotid tissue just around the capsule of the neoplasm (7). Mobility of the lesion, a thin covering of tumor capsule, and dimensions of the damage large enough for digital manipulation during operation are supportive elements for choosing this technique (7). Albeit, surgical experience is recommended since there is a high risk of mistakes (7). This approach seems to be useful also in deep lobe parotid neoplasms (4).

As regards the steps of the operation, an incision is conducted for at least $1 \mathrm{~cm}$ surrounding the damage and a flap is raised; the tumor is separated from the parotid gland while parotid fascia is lifted away with the use of artery clips; then, a careful removal is recommended in order to prevent facial verve; resorbable sutures and a small drain are used for closing the wound which decrease the danger of Frey's syndrome; 
finally, the patients are able to return home the next day (4). Nerve monitor has been suggested for decreasing risk of damaging facial nerve without success (4). No adverse events are reported (including permanent nerve weakness) except for temporary weakness in less than $7 \%$ of cases (4).

Although ECD is supported to result in a lower risk of facial nerve injury compared to SP, some authors claim that narrow surgical margins after ECD have been related to recurrence $(4,9)$. In such cases, a combined use of chemotherapy and radiotherapy could be an alternative as long as these patients are systematically healthy (9). Recurrence rate is reported to be similar between ECD and SP since ECD is conducted properly without leaving tumor remnants (4).

\section{Other therapeutic techniques}

Transoral endoscopic and robotic methods as an alternative for salivary gland swellings should be carefully evaluated (2).

\section{Conservation of facial nerve}

Prevention of the facial nerve has been the ultimate operation goal for years mainly during benign or intermediate-grade malignant lesions removals (10). Albeit, when aggressive high-grade malignant swellings are present, it is often required to sacrifice the facial nerve $(10,11)$. More specifically, the more invasive is the approach the higher risk of facial nerve paresis is probable $(1,11)$. In any case, the repair of the injured facial nerve is essential as to rehabilitate facial tone and symmetry at rest, voluntary and spontaneous facial movements, and to reduce the cosmetic and functional adverse event following facial paralysis (10).

\section{Frey's syndrome}

Frey's syndrome (gustatory sweating) may occur after parotid surgery and depends on the parasympathetic nerves which supply the gland redirecting and innervating the skin swea glands, leading in sweating before or during mastication (4). A direct outcome of this syndrome is the worsening of patients' quality of life (4). As it has already mentioned, ECD leads in much lower rate of Frey's syndrome incindence (4).

\section{Adjuvant therapy - Radiation and chemotherapy}

Generally, thorough surgical excision constitutes the treatment of choice[8]. However, radiation or combined application of radiation and chemotherapy could be concerned as useful adjuvant therapy following surgical operation (12). In the past, radiation was not recommended as curative therapeutic choice due to the radioresistance of malignant salivary gland lesions $(13,14)$. Today, it is clear that radiotherapy has favorable outcomes in relation to locoregional control but cannot be effective to distant metastasis $(1,8,13,15)$. This element supports the less invasive surgical procedures in cases of neoplasms with high risk of poor aesthetic or functional outcome postoperatively (15).

The effectiveness of chemotherapy in salivary gland tumors has been a field of controversy with the supporters claiming a small improvement in neoplasms with slow progress $(1,8)$. In any case, it essential each patient who is candidate for chemotherapy to be well informed not only about the benefits but also 
about the toxicities related to this treatment (16). As a result, chemotherapy should be applied only when surgery and radiation therapies are no longer effective for the patient (16).

\section{Conclusion}

In conclusion, it evident that the treatment of choice of salivary gland tumors is always individual and depends on clinical and hystopathologiclal characteristics on the tumor process. The use of radiation and chemotherapy in well selected patients leads in improvement of locoregional control without controlling distant metastasis.

Table 1. Current classification of salivary gland tumors[3].

\begin{tabular}{|l|l|}
\hline \multicolumn{1}{|c|}{ Type } & \\
\hline$T_{\mathrm{x}}$ & Difficulty to be assessed. \\
\hline $\mathrm{T}_{0}$ & No evidence for the presence of primary neoplasm. \\
\hline $\mathrm{T}_{1}$ & Less than 2cm in size \& no extraparenchymal extension. \\
\hline $\mathrm{T}_{2}$ & Between 2 and 4cm in size \& no extraparenchymal extension. \\
\hline $\mathrm{T}_{3}$ & More than 4cm \& extraparenchymal extension. \\
\hline $\mathrm{T}_{4 \mathrm{a}}$ & Invasion of skin, mandible, ear canal and/or facial nerve. \\
\hline $\mathrm{T}_{4 \mathrm{~b}}$ & Invasion of skull base and/or pterygoid plates and/or enclosed carotid artery. \\
\hline
\end{tabular}

Table 2. Current therapeutic approaches of management of salivary gland tumors.

\begin{tabular}{|l|l|l|}
\hline \multicolumn{1}{|c|}{ Surgery } & \multicolumn{1}{|c|}{ Neck surgery } & \multicolumn{1}{|c|}{ Adjuvant therapy } \\
\hline 1.partial parotidectomy & 1.none & 1.radiotherapy \\
\hline 2.total parotidectomy & 2.selective neck dissection & 2.concurrent chemoradiotherapy \\
\cline { 1 - 1 } $\begin{array}{l}\text { 3.total parotidectomy \& nerve } \\
\text { sacrifice }\end{array}$ & $\begin{array}{l}\text { 3.modified radical neck } \\
\text { dissection }\end{array}$ & \\
$\begin{array}{l}\text { 4.superficial parotidectomy \& } \\
\text { radiotherapy }\end{array}$ & & \multirow{2}{*}{ Questioned methods } \\
\cline { 1 - 1 } 5.trancervical approach & & \\
\cline { 1 - 1 } 6.extracapsular dissection & & 1.transoral endoscopic approach \\
7.rhytidectomy & & \\
\hline
\end{tabular}




\section{References}

1. Scianna JM, Petruzzelli G J. Contemporary management of tumors of the salivary glands. Curr Oncol Rep 2007;9:2:134-138.

2. Eisele DW, Richmon JD. Contemporary evaluation and management of parapharyngeal space neoplasms. J Laryngol Otol 2013;127: 6:550-555.

3. Sood S, McGurk M, Vaz F. Management of Salivary Gland Tumours: United Kingdom National Multidisciplinary Guidelines. J Laryngol Otol 2016; 130: S142-S149.

4. Brennan PA, Ammar M, Matharu J. Contemporary management of benign parotid tumours - the increasing evidence for extracapsular dissection. Oral Dis 2017; 23:1:18-21.

5. Said-Al-Naief N, Sciandra K, Gnepp DR. Moderately Differentiated Neuroendocrine Carcinoma (Atypical Carcinoid) of the Parotid Gland: Report of Three Cases with Contemporary Review of Salivary Neuroendocrine Carcinomas. Head Neck Pathol 2013; 7, 3, 295-303.

6. Mifsud M, Sharma S, Leon M, et al.Salivary Duct Carcinoma of the Parotid. Otolaryngol Neck Surg 2016;154, 6, 1041-1046.

7. Witt RL.The Significance of the Margin in Parotid Surgery for Pleomorphic Adenoma. Laryngoscope 2002; 112,12,2141-2154.

8. Yang S, Li L, Zeng $\mathrm{M}$, et al. Myoepithelial carcinoma of intraoral minor salivary glands: A clinicopathological study of 7 cases and review of the literature. Oral Surgery Oral Med Oral Pathol Oral Radiol Endodontology, 2010; 110, 1,85-93.

9. Gil Z, Fliss DM. Contemporary management of head and neck cancers. Isr Med Assoc J 2009; 11: 5:296-300.

10. Biglioli $\mathrm{F}$ et al. Thoracodorsal nerve graft for reconstruction of facial nerve branching. J. CranioMaxillofacial Surg 2014; 42:1: e8-e14.

11. Guntinas-Lichius O, Genther DJ, Byrne PJ. Facial Reconstruction and Rehabilitation. Adv Otorhinolaryngol 2016; 78:120-131.

12. Chiosea SI. Molecular Characterization of Apocrine Salivary Duct Carcinoma. Am J Surg Pathol 2015;39:744-752.

13. T. Luu et al. Major salivary gland tumors: Treatment results and prognostic factors. Laryngoscope 1986; 96:10: 1139-1144.

14. Jegadeesh $\mathrm{N}$ et al.Outcomes and prognostic factors in modern era management of major salivary gland cancer. Oral Oncol 2015;51:8:770-777.

15. Terhaard $\mathrm{C} \mathrm{H} \mathrm{J} \mathrm{et} \mathrm{al.} \mathrm{The} \mathrm{role} \mathrm{of} \mathrm{radiotherapy} \mathrm{in} \mathrm{the} \mathrm{treatment} \mathrm{of} \mathrm{malignant} \mathrm{salivary} \mathrm{gland} \mathrm{tumors.}$ Int J Radiat Oncol 2005: 61: 1:103-111.

16. Creagan ET, Woods JE, RubinJ, et al. Cisplatin based chemotherapy for neoplasms arising from salivary glands and contiguous structures in the head and neck. Cancer 1988;62:11: 2313-2319.

\section{Corresponding author:}

Assya Krasteva

Faculty of Dental Medicine

Department of Diagnostic Imaging and Oral Diagnostic

Medical University of Sofia 